Article

\title{
Prevalence of Peripheral Arterial Disease and Associated Vascular Risk Factors in 65-Years-Old People of Northern Barcelona
}

\author{
Gabriela Gonçalves-Martins ${ }^{1,2}$, Daniel Gil-Sala ${ }^{1, * \mathbb{D}}$, Cristina Tello-Díaz ${ }^{1}$, Xavier Tenezaca-Sari ${ }^{1}$ (D), \\ Carlos Marrero ${ }^{1}$, Teresa Puig ${ }^{3,4}$, Raquel Gayarre ${ }^{5,6}{ }^{\mathbb{D}}$, Joan Fité ${ }^{7}$ and Sergi Bellmunt-Montoya ${ }^{1,2}$
}

1 Angiology, Vascular and Endovascular Surgery Department, Hospital Vall d'Hebron, 08035 Barcelona, Spain; ggoncalves@vhebron.net (G.G.-M.); cristina.tello11@gmail.com (C.T.-D.); atenezaca@vhebron.net (X.T.-S.); cmarrero@vhebron.net (C.M.); sbellmunt@vhebron.net (S.B.-M.)

2 Departament de Cirurgia, Universitat Autònoma de Barcelona (UAB), 08035 Barcelona, Spain

3 Department of Clinical Epidemiology and Public Health, Hospital de la Santa Creu i Sant Pau. IIB Sant Pau, 08041 Barcelona, Spain; tpuig@santpau.cat

4 Deparment of Pediatrics, Obstetrics and Gynecology and Preventive Medicine, Universitat Autònoma de Barcelona (UAB), CIBERCV. 08035, Barcelona, Spain

5 Primary Care Department, Institut Català de la Salut, 08041 Barcelona, Spain; rgayarre@gencat.cat

6 Departament de Medicina, Unitat Docent Sant Pau, Universitat Autònoma de Barcelona (UAB), 08025 Barcelona, Spain

check for updates

Citation: Gonçalves-Martins, G.; Gil-Sala, D.; Tello-Díaz, C.;

Tenezaca-Sari, X.; Marrero, C.; Puig, T.; Gayarre, R.; Fité, J.;

Bellmunt-Montoya, S. Prevalence of Peripheral Arterial Disease and Associated Vascular Risk Factors in 65-Years-Old People of Northern Barcelona. J. Clin. Med. 2021, 10, 4467. https://doi.org/10.3390/jcm10194467

Academic Editors:

Christian-Alexander Behrendt and Frederik Peters

Received: 29 August 2021

Accepted: 24 September 2021

Published: 28 September 2021

Publisher's Note: MDPI stays neutral with regard to jurisdictional claims in published maps and institutional affiliations.

Copyright: (c) 2021 by the authors. Licensee MDPI, Basel, Switzerland. This article is an open access article distributed under the terms and conditions of the Creative Commons Attribution (CC BY) license (https:/ / creativecommons.org/licenses/by/ $4.0 /)$.
7 Angiology, Vascular and Endovascular Surgery Department, Hospital de la Santa Creu i Sant Pau, Institut de Recerca Biomèdica de Sant Pau (IIB-Sant Pau), CIBERCV, 08041 Barcelona, Spain; jfite@santpau.cat * Correspondence: dgil@vhebron.net; Tel.: +34-93-2746131

\begin{abstract}
Objective: To determine the prevalence and risk factors associated with peripheral arterial disease (PAD) in Northern Barcelona at 65 years of age. Methods: A single-center, cross-sectional study, including males and females 65 years of age, health care cardholders of Barcelona Nord. PAD was defined as an ankle-brachial index $(\mathrm{ABI})<0.9$. Attending subjects were evaluated for a history of common cardiovascular risk factors. A REGICOR score was obtained, as well as a physical examination and anthropometric measurements. Results: From November 2017 to December 2018, 1174 subjects were included: $479(40.8 \%)$ female and $695(59.2 \%)$ male. Overall prevalence of PAD was 6.2\% (95\%CI: 4.8-7.6\%), being 7.9\% (95\%CI: 5.9-9.9\%) in males and 3.8\% (95\%CI: $2.1-5.5 \%$ ) in females. An independent strong association was seen in male smokers and diabetes, with ORs pf 7.2 (95\% CI: 2.8-18.6) and 1.8 (95\% CI: 1.0-3.3), respectively, and in female smokers and hypertension, with ORs of 5.2 (95\% CI: 1.6-17.3) and 3.3 (95\% CI: 1.2-9.0). Male subjects presented with higher REGICOR scores $(p<0.001)$. Conclusion: Higher-risk groups are seen in male subjects with a history of smoking and diabetes and female smokers and arterial hypertension, becoming important subgroups for our primary healthcare centers and should be considered for ABI screening programs.
\end{abstract}

Keywords: peripheral arterial disease; cardiovascular risk factors; asymptomatic; ankle-brachial index; prevalence; screening

\section{Introduction}

Peripheral arterial disease (PAD) is the manifestation of atherosclerotic disease in the lower extremities, resulting in narrowing of the blood vessels and diminishing blood flow to the limbs. It is the third leading cause of cardiovascular pathology after coronary and cerebrovascular disease and an important indicator of cardiovascular risk [1]. The mere presence of PAD can indicate a 6.6-fold increased risk of cardiovascular disease association [2], becoming, therefore, an important indicator of the coexistence of other cardiovascular pathologies.

Associated risk factors are common for cardiovascular diseases, such as smoking, diabetes, dyslipidemia and hypertension, among others. Active smoking has the strongest 
association, doubling the risk of PAD in these patients [3]. Therefore, early diagnosis and treatment of modifiable risk factors is an important tool in preventing and treating this disease, consequently lowering associated cardiovascular disease morbidity and mortality.

The ankle-brachial index (ABI) is an effective, easy and first-line noninvasive method in screening PAD [4-6], with an overall $69-79 \%$ sensitivity and $83-99 \%$ specificity for detecting $>50 \%$ arterial stenosis [7]. Despite the feasibility of this technique, this pathology is well known to be underdiagnosed and undertreated [4,5].

In the United States, 8 to 12 million Americans suffer from PAD, with an overall prevalence of 3-10\%, which increases to almost $50 \%$ in those greater than 85 years old $[8,9]$. As for Europe, prevalence as high as $17.8 \%$ (99\% CI: 16.84-18.83\%) was observed in some studies, especially in the northern region [10].

PAD is also influenced by gender and age, predominantly being associated with the male gender. Age is also an important factor, with a $20 \%$ increase observed in those older than $75[11,12]$. In a global aging society, this can present an important social and economic burden.

To our knowledge, most studies focused on the prevalence of PAD performed in our region were inclusive of younger age groups, some including ages as young as 35 years old $[13,14]$. This perhaps explains the low prevalence results achieved in these studies. Due to the important variety of factors that influence the prevalence of this pathology, age being one of them, we decided to perform a study including individuals of 65 years old in order to analyze if an increase of prevalence was seen in these groups and, as such, evaluate if there were differences in our gender groups with this increase in age, as well as associated risk factors. This way perhaps allows pinpointing a target group that requires a more detailed initial evaluation and opportunistic screening (with $A B I$ ) in our primary care centers in order to prevent and/or diagnose PAD.

Thus, the aim of our study was to determine the prevalence of peripheral arterial disease through ABI in 65-year-old men and women in Northern Barcelona (Spain), as well as its associated risk factors.

\section{Materials and Methods}

This is a single-center, populational-based, cross-sectional study, part of a larger pilot screening program evaluating abdominal aorta aneurysm [15]. The protocol was approved by the ethics committee of Hospital Vall d'Hebron with code PR(AG)221/2017.

\subsection{Patient Selection}

The study was carried out in a health-integrated area AIS-Barcelona Nord, with a population base of 400,000 inhabitants, which corresponds to the area of treatment of our center. Inclusion criteria were all noninstitutionalized males and females of 65 years of age during the time of recruitment who were health care cardholders and resided for at least 6 months in the referred area.

Contact information of the population was authorized and obtained through our public health census registry, and all eligible subjects received invitation letters informing them of the present study and tests to be performed. Those with incorrect postal data, terminal disease and deceased subjects were considered ineligible (Figure 1).

Nonresponders to the first letter were invited again within 1 month. Those who agreed to participate were scheduled an appointment at our Vascular Lab Center with one of five trained vascular surgeon residents. Participants were contacted $48 \mathrm{~h}$ before the appointment to confirm attendance. Those who did not show up for the scheduled appointment after 3 occasions, those who did not wish to participate and subjects who did not respond to both invitation letters were excluded. 


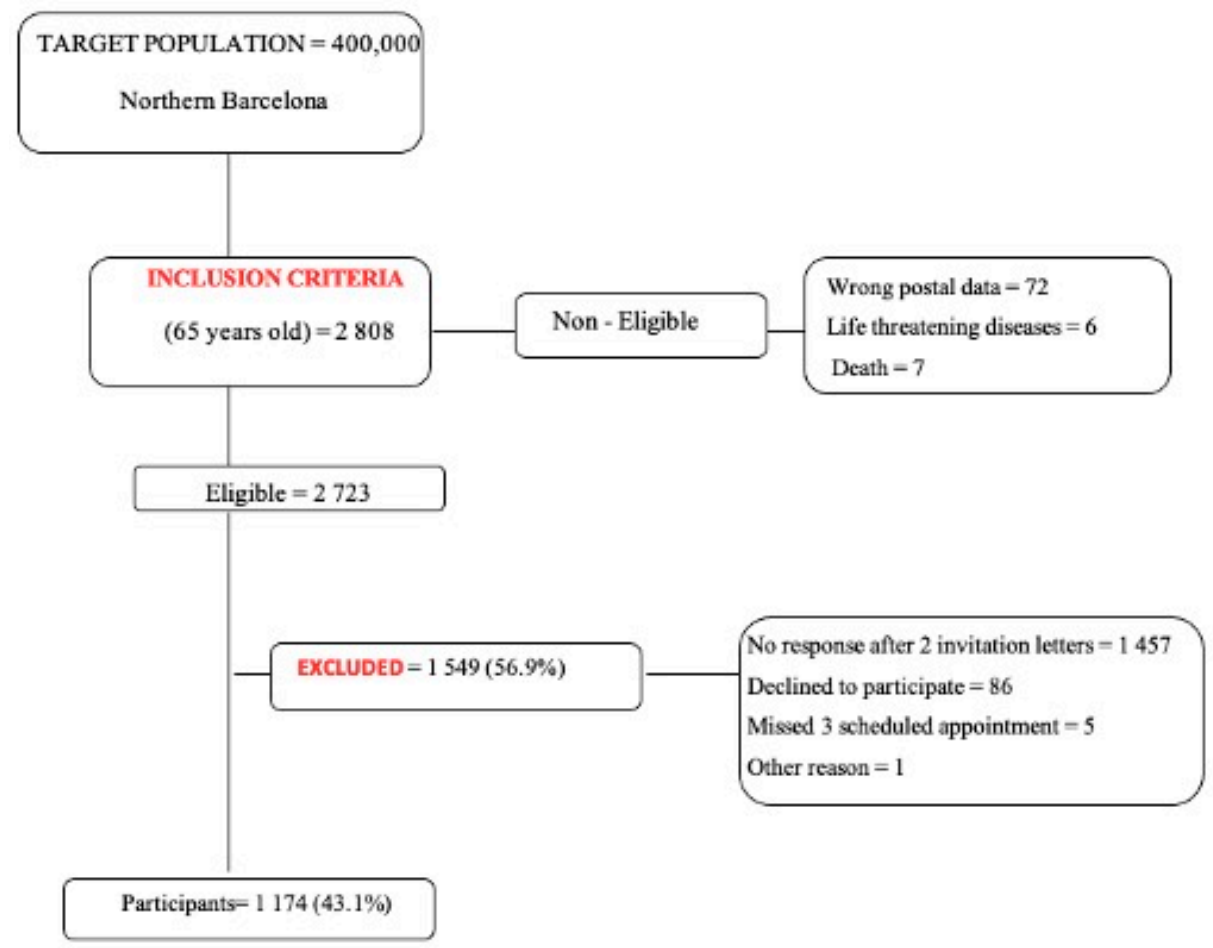

Figure 1. Flowchart of initial inclusion description.

\subsection{Variables Evaluated}

After arrival, prior to questioning, a signed consent form was required from each participant. All subjects were questioned for a history of risk factors, such as: cigarette smoking: active, former (more than a year without smoking) or nonsmoker; hypertension, defined as systolic blood pressure $\geq 140 / 90 \mathrm{mmHg}$ or subjects under antihypertensive treatment; dyslipidemia, defined as one or all of total blood cholesterol $>200 \mathrm{mg} / \mathrm{dL}$, lowdensity lipoprotein (LDL) cholesterol $>100 \mathrm{mg} / \mathrm{dL}$ or triglycerides $>150 \mathrm{mg} / \mathrm{dL}$ or subjects under treatment for dyslipidemia; diabetes, defined as those diagnosed and/or under antidiabetic treatment; history of cerebrovascular disease (previous stroke or transient ischemic cerebral accident); history of cardiac ischemia (diagnosed coronary artery disease or previous myocardial infarction); chronic renal disease (glomerular filtration $<60 \mathrm{~mL} / \mathrm{min}$ ) and history of any aortic aneurismatic disease. Anthropometric measurements were also recorded: height $(\mathrm{m})$, weight $(\mathrm{kg})$, waist circumference $(\mathrm{cm})$ and body mass index (BMI). A REGICOR score [16] was determined for each individual to evaluate primary prevention, discarding those subjects who had already presented a previous cardiovascular event. Cholesterol levels and blood pressure required for scoring were obtained through medical records. The REGICOR (Girona Heart Registry) research group focuses on ischemic heart disease and associated risk factors in order to improve preventive strategies. This score assesses the risk of presenting a cardiovascular event in the following 10 years through a computer-generated calculation that evaluated age, sex, history of smoking, presence of diabetes and blood pressure, total cholesterol and high-density lipoprotein (HDL) levels. Low risks are considered to be scores $<5 \%$, moderate risk between 5 and $9 \%$ and high-risk subjects $>10 \%$.

\subsection{Physical Assessment}

To complete the assessment, a physical exam was performed, evaluating distal pulses through palpation. The presence of a pulse in one or both tibial arteries was considered adequate. Afterward, an ABI was performed in a standard manner [7]: after a $5 \mathrm{~min}$ rest, with the subjects in a supine position, systolic blood pressure was measured at the level of the posterior and anterior tibial arteries of both lower extremities and at the level of the brachial artery of both upper extremities, with a continuous wave Doppler probe 
(Flowsoft 7 Angiolab1 Spead Doppler Systeme, Kehl, Germany) and an arterial pressure cuff that was placed just above the malleoli. ABI was calculated through the ratio of systolic pressure of the tibial arteries to the highest brachial systolic pressure. ABI of both extremities was recorded. Those with an ABI $<0.9$ in one or both lower extremities were considered pathological with an indication of PAD. All ABI > 1.4 were registered. In the case that a patient presented with critical limb ischemia, considered as rest pain or presence of ischemic lesions, they were sent to the vascular emergency room for evaluation. At the end of each assessment, the patient was given a signed report indicating the result of their ABI exam, and if the exam was pathological, recommendations of the modifiable risk factors were given in order to prevent the progression of PAD.

For better analysis and interpretation of our results, we reviewed common risk factors among our study participants and those obtained from the official health department database, 2018 Catalan Health Survey "Enquesta Catalunya de Salut 2018" (ESCA) (ages 65-74 years old), and our primary care clinics (65 years old), with previous authorization from our health department.

\subsection{Statistical Analysis}

Descriptive analysis was performed, and prevalence of PAD was obtained with the 95\% confidence interval. Dichotomous variables were analyzed through Pearson's chi-square test or Fisher's exact test. Logistic regression models were used to obtain independent associated risk factors for PAD that were previously significant in the bivariate analysis. Interaction effect was also evaluated among these risk factors. Statistical significance results were considered for a value of $p<0.05$. Statistical analysis was performed through IBM SPSS Statistics for Windows, version 22.0 (IBM Corp., Armonk, NY, USA).

\section{Results}

From November 2017 to December 2018, a total of 2808 subjects were 65 years old during the time of recruitment. A total of 1174 subjects were finally included, 695 (59.2\%) male and $479(40.8 \%)$ female. Total participation rate was $43.1 \%$, with higher participation from females $(46.7 \%)$ than males $(40.9 \%)$. A flow chart of initial screening with main results separated by sex is shown in Figure 2.

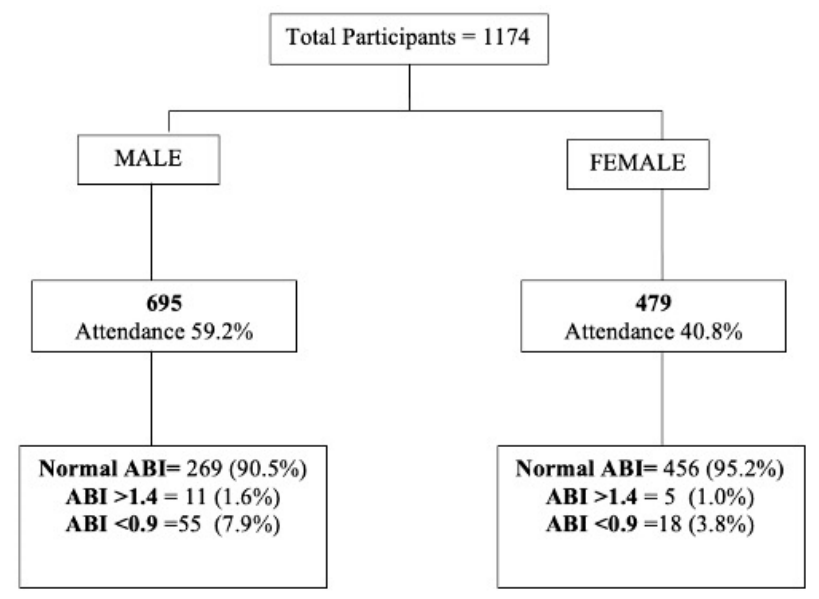

Figure 2. Flowchart screening results.

The overall prevalence of PAD was 6.2\% (95\% CI: $4.8-7.6 \%)$, male $7.9 \%$ (95\% CI: 5.9-9.9\%) and female 3.8\% (95\% CI: 2.1-5.5\%), most of them corresponding to the Fontaine I classification $(n=53,72.6 \%), 20.5 \%$ IIA $(n=15), 5.5 \%$ IIB $(n=4)$ and $1.4 \%(n=1)$. The difference in prevalence between male and female was statistically significant $(p=0.006)$. Male subjects presented at higher risk for PAD, with an OR of 2.2 (CI: 1.3-3.8).

There was a total of $18 \mathrm{ABI}>1.4$, of which two of these had contralateral ABIs $<0.9$ considered PAD. Of the other 16 ABIs $>1.4$ (6 were diabetic subjects, and none had chronic 
renal disease), all subjects except for one had palpable distal pulses and none referred to intermittent claudication.

Diabetes male subjects were more likely to have $\operatorname{PAD}(p=0.017)$ and female subjects hypertension $(p=0.026)$ and waist circumference $(p=0.018)$. No association was seen with a family history of abdominal aortic aneurysm, probably due to the small number of cases detected. Table 1 compares risk factors presented in PAD and non-PAD divided by gender.

Table 1. Relationship between PAD and risk factors divided by gender. Logistic regression model with OR and CI 95\% of the potential variables associated with PAD.

\begin{tabular}{|c|c|c|c|c|c|c|c|c|c|c|}
\hline & \multicolumn{5}{|c|}{ Male } & \multicolumn{5}{|c|}{ Female } \\
\hline & $\begin{array}{l}\text { Non-PAD } \\
(n=640)\end{array}$ & $\begin{array}{c}\text { PAD } \\
(n=55)\end{array}$ & $\begin{array}{c}\text { Total } \\
(n=695)\end{array}$ & $\begin{array}{l}\text { OR* }(95 \% \\
\left.\text { CI: }{ }^{* *}\right)\end{array}$ & $p^{\mathrm{a}}$ & $\begin{array}{l}\text { Non-PAD } \\
(n=461)\end{array}$ & $\begin{array}{c}\text { PAD } \\
(n=18)\end{array}$ & $\begin{array}{c}\text { Total } \\
(n=479)\end{array}$ & $\begin{array}{l}\text { OR }(95 \% \\
\left.\text { CI: }{ }^{* *}\right)\end{array}$ & $p^{\mathrm{a}}$ \\
\hline Non-smoker & $\begin{array}{c}237 \\
(37.0 \%)\end{array}$ & $\begin{array}{c}6 \\
(10.9 \%)\end{array}$ & $\begin{array}{c}243 \\
(35.0 \%)\end{array}$ & 1 & $<0.001$ & $\begin{array}{c}320 \\
(69.4 \%)\end{array}$ & $\begin{array}{c}7 \\
(38.9 \%)\end{array}$ & $\begin{array}{c}327 \\
(68.3 \%)\end{array}$ & 1 & 0.02 \\
\hline Former smoker & $\begin{array}{c}299 \\
(46.7 \%)\end{array}$ & $\begin{array}{c}29 \\
(52.7 \%)\end{array}$ & $\begin{array}{c}328 \\
(47.2 \%)\end{array}$ & $3.8(1.6-9.40)$ & 0.003 & $\begin{array}{c}96 \\
(20.8 \%)\end{array}$ & $\begin{array}{c}6 \\
(33.3 \%)\end{array}$ & $\begin{array}{c}102 \\
(21.3 \%)\end{array}$ & $\begin{array}{c}2.9 \\
(0.9-8.7)\end{array}$ & 0.065 \\
\hline Active smoker & $\begin{array}{c}104 \\
(16.3 \%)\end{array}$ & $\begin{array}{c}20 \\
(36.4 \%)\end{array}$ & $\begin{array}{c}124 \\
(17.8 \%)\end{array}$ & $\begin{array}{c}7.6 \\
(3.0-19.5)\end{array}$ & $<0.001$ & $\begin{array}{c}45 \\
(9.8 \%)\end{array}$ & $\begin{array}{c}5 \\
(27.8 \%)\end{array}$ & $\begin{array}{c}50 \\
(10.4 \%)\end{array}$ & $\begin{array}{c}5.1 \\
(1.5-16.7)\end{array}$ & 0.007 \\
\hline \multicolumn{11}{|c|}{ Cardiovascular risk factors n (\%) } \\
\hline Diabetes & $\begin{array}{c}141 \\
(22.0 \%)\end{array}$ & $\begin{array}{c}20 \\
(36.4 \%)\end{array}$ & $\begin{array}{c}161 \\
(23.2 \%)\end{array}$ & $\begin{array}{c}2.0 \\
(1.1-3.6)\end{array}$ & 0.017 & $\begin{array}{c}43 \\
(9.3 \%)\end{array}$ & $\begin{array}{c}4 \\
(22.2 \%)\end{array}$ & $\begin{array}{c}47 \\
(9.8 \%)\end{array}$ & $\begin{array}{c}2.8 \\
(0.9-8.8)\end{array}$ & 0.083 \\
\hline Dyslipidemia & $\begin{array}{c}293 \\
(45.8 \%)\end{array}$ & $\begin{array}{c}26 \\
(47.3 \%)\end{array}$ & $\begin{array}{c}319 \\
(45.9 \%)\end{array}$ & $\begin{array}{c}1.1 \\
(0.6-1.8)\end{array}$ & 0.831 & $\begin{array}{c}215 \\
(46.6 \%)\end{array}$ & $\begin{array}{c}10 \\
(55.6 \%)\end{array}$ & $\begin{array}{c}225 \\
(47.0 \%)\end{array}$ & $\begin{array}{c}1.4 \\
(0.6-3.7)\end{array}$ & 0.459 \\
\hline Hypertension & $\begin{array}{c}340 \\
(53.1 \%)\end{array}$ & $\begin{array}{c}28 \\
(50.9 \%)\end{array}$ & $\begin{array}{c}368 \\
(52.9 \%)\end{array}$ & $\begin{array}{c}0.9 \\
(0.5-1.6)\end{array}$ & 0.752 & $\begin{array}{c}181 \\
(39.3 \%)\end{array}$ & $\begin{array}{c}12 \\
(66.7 \%)\end{array}$ & $\begin{array}{c}193 \\
(40.3 \%)\end{array}$ & $\begin{array}{c}3.1 \\
(1.1-8.4)\end{array}$ & 0.026 \\
\hline $\begin{array}{l}\text { Chronic Renal } \\
\text { Disease }\end{array}$ & $\begin{array}{c}37 \\
(5.8 \%)\end{array}$ & $\begin{array}{c}3 \\
(5.5 \%)\end{array}$ & $\begin{array}{c}40 \\
(5.8 \%)\end{array}$ & $\begin{array}{c}0.9 \\
(0.3-3.2)\end{array}$ & 0.920 & $\begin{array}{c}8 \\
(1.7 \%)\end{array}$ & $\begin{array}{c}0 \\
(0 \%)\end{array}$ & $\begin{array}{c}8 \\
(1.7 \%)\end{array}$ & 0 & 0.9 \\
\hline Cardiac Ischemia & $\begin{array}{c}59 \\
(9.2 \%)\end{array}$ & $\begin{array}{c}9 \\
(16.4 \%)\end{array}$ & $\begin{array}{c}68 \\
(9.8 \%)\end{array}$ & $\begin{array}{c}1.9 \\
(0.9-4.1)\end{array}$ & 0.092 & $\begin{array}{c}6 \\
(1.3 \%)\end{array}$ & $\begin{array}{c}1 \\
(5.6 \%)\end{array}$ & $\begin{array}{c}7 \\
(1.5 \%)\end{array}$ & $\begin{array}{c}4.5 \\
(0.5-39.1)\end{array}$ & 0.177 \\
\hline $\begin{array}{c}\text { Cerebrovascular } \\
\text { Events }\end{array}$ & $\begin{array}{c}31 \\
(4.8 \%)\end{array}$ & $\begin{array}{c}3 \\
(5.5 \%)\end{array}$ & $\begin{array}{c}34 \\
(4.9 \%)\end{array}$ & $\begin{array}{c}1.1 \\
(0.3-3.8)\end{array}$ & 0.840 & $\begin{array}{c}16 \\
(3.5 \%)\end{array}$ & $\begin{array}{c}0 \\
(0 \%)\end{array}$ & $\begin{array}{c}16 \\
(3.3 \%)\end{array}$ & 0 & 0.9 \\
\hline \multicolumn{11}{|c|}{ Anthropometric measurements mean (SD) } \\
\hline $\begin{array}{l}\text { Waist Circumference } \\
\qquad(\mathrm{cm})\end{array}$ & $\begin{array}{c}102.4 \\
(\mathrm{SD} 11.0)\end{array}$ & $\begin{array}{c}103.6 \\
(\mathrm{SD} 9.4)\end{array}$ & $\begin{array}{c}102.5 \\
(\mathrm{SD} 10.9)\end{array}$ & $\begin{array}{c}1.01 \\
(0.99-1.04)\end{array}$ & 0.410 & $\begin{array}{c}95.1 \\
(\mathrm{SD} 12.1)\end{array}$ & $\begin{array}{c}102.4 \\
(\mathrm{SD} 15.9)\end{array}$ & $\begin{array}{c}95.3 \\
\text { (SD 12.3) }\end{array}$ & $\begin{array}{c}1.05 \\
(1.01-1.09)\end{array}$ & 0.018 \\
\hline
\end{tabular}

Data are expressed as $n$ (\%) or mean (standard deviation); * OR: odds ratio; ** CI: confidence interval; ${ }^{*}$ Wald test's $p$-values of logistic regression.

A multilogistic regression model was performed from the significant bivariate analysis variables. In our male model, an independent association between PAD and smoking was shown. PAD in male subjects was higher in ex-smokers, with an OR of 3.5 (95\% CI: 1.4-8-7) and in active smokers 7.2 (95\% CI: 2.8-18.6) in comparison with nonsmokers. An association with diabetes and PAD was also seen in our male participants, with an OR of 1.8 (95\% CI: $1.0-3.3)$

As for our female model, an association was also seen in relation to PAD and smoking, with an OR of 3.1 (95\% CI: 1.0-9.6) in ex-smokers and 5.2 (95\% CI: 1.6-17.3) in active smokers compared with nonsmokers. PAD was also higher in those female subjects with hypertension, with an OR of 3.3 (95\% CI: 1.2-9.0) (Table 2). No significant association was seen between hypertension and PAD in male subjects nor diabetes and PAD in our female subjects. Therefore, no interaction effect was observed among these variables, and no mediation effect was observed with gender.

Table 2. Multiple logistic regression analysis of PAD risk.

\begin{tabular}{cccc}
\hline & Male & \multicolumn{2}{c}{ Female } \\
\hline Variables & OR (95\% CI) & Variables & OR (95\% CI) \\
\hline Non-smokers & 1 & Non-smokers & 1 \\
\hline Former Smokers & $3.5(1.4-8.7)$ & Former Smokers & $3.1(1.0-9.6)$ \\
\hline Active Smokers & $7.2(2.8-18.6)$ & Active Smokers & $5.2(1.6-17.3)$ \\
\hline Diabetes & $1.8(1.0-3.3)$ & High blood pressure & $3.3(1.2-9.0)$ \\
\hline
\end{tabular}

CI: confidence interval. OR: odds ratio. 
Discarding 157 subjects who had already presented a previous cardiovascular event, 1013 subjects were classified according to their cardiovascular risk at 10 years with the REGICOR scale. In this regard, $51.4 \%(n=500)$ of our population were at low risk of developing a cardiovascular event in the following 10 years, $40.4 \%(n=393)$ were at moderate risk and $8.1 \%(n=79)$ were at a high-risk score. Four subjects were excluded from REGICOR analysis due to missing data. Male subjects presented with higher REGICOR scores $(p<0.001)$, putting them at a higher risk for developing a cardiovascular event in the following 10 years compared to our female subjects. Table 3 illustrates the REGICOR score in our general population divided by sex and PAD.

Table 3. REGICOR results in general population separated by sex and PAD.

\begin{tabular}{ccccccc}
\hline & \multicolumn{3}{c}{ Non-PAD } & \multicolumn{3}{c}{ PAD } \\
\hline & $\begin{array}{c}\text { TOTAL } \\
(\boldsymbol{n}=\mathbf{9 7 2})\end{array}$ & $\begin{array}{c}\text { MALE } \\
(\boldsymbol{n}=\mathbf{5 4 3})\end{array}$ & $\begin{array}{c}\text { FEMALE } \\
(\boldsymbol{n}=\mathbf{4 2 9 )}\end{array}$ & $\begin{array}{c}\text { TOTAL } \\
(\boldsymbol{n}=\mathbf{4 1})\end{array}$ & $\begin{array}{c}\text { MALE } \\
(\boldsymbol{n}=\mathbf{2 7})\end{array}$ & $\begin{array}{c}\text { FEMALE } \\
(\boldsymbol{n}=\mathbf{1 4})\end{array}$ \\
\hline $\begin{array}{c}\text { Low risk } \\
<5 \%\end{array}$ & $\begin{array}{c}51.4 \% \\
(500)\end{array}$ & $\begin{array}{c}33.1 \% \\
(180)\end{array}$ & $\begin{array}{c}74.6 \% \\
(320)\end{array}$ & $\begin{array}{c}48.8 \% \\
(20)\end{array}$ & $\begin{array}{c}40.7 \% \\
(11)\end{array}$ & $\begin{array}{c}64.3 \% \\
(9)\end{array}$ \\
\hline $\begin{array}{c}\text { Moderate } \\
\text { risk }\end{array}$ & $40.4 \%$ & $\begin{array}{c}54.1 \% \\
5-9 \%\end{array}$ & $\begin{array}{c}23.1 \% \\
(393)\end{array}$ & $\begin{array}{c}41.5 \% \\
(294)\end{array}$ & $\begin{array}{c}44.4 \% \\
(12)\end{array}$ & $\begin{array}{c}35.7 \% \\
(5)\end{array}$ \\
\hline $\begin{array}{c}\text { High risk } \\
>10 \%\end{array}$ & $\begin{array}{c}(7.1 \% \\
(79)\end{array}$ & $\begin{array}{c}12.7 \% \\
(69)\end{array}$ & $\begin{array}{c}2.3 \% \\
(10)\end{array}$ & $\begin{array}{c}9.8 \% \\
(4)\end{array}$ & $\begin{array}{c}14.8 \% \\
(4)\end{array}$ & $\begin{array}{c}0 \% \\
(0)\end{array}$ \\
\hline
\end{tabular}

Pearson's chi-square analysis; 4 subjects without REGICOR.

Characteristics of our 1174 studied men and women are best shown in Table 4. Overall, fewer women were smokers $(p<0.001)$, and our male subjects had higher percentages of diabetes $(p<0.001)$, high blood pressure $(p<0.001)$, chronic renal insufficiency $(p=0.001)$ and cardiac ischemia $(p<0.001)$, presenting with more risk factors than our female participants. Regarding the history of aneurysmal disease, $3 \%(n=35)$ had a family history of abdominal aneurismatic disease, with 26 of them being a first-degree family member.

Table 4. Means and proportions of selected characteristics in the screened population separated by sex.

\begin{tabular}{|c|c|c|c|}
\hline & $\begin{array}{c}\text { Total } \\
(n=1174)\end{array}$ & $\begin{array}{c}\text { Male } \\
(n=695)\end{array}$ & $\begin{array}{c}\text { Female } \\
(n=479)\end{array}$ \\
\hline \multicolumn{4}{|c|}{ Cardiovascular Risk Factors\% (N) } \\
\hline Non-smokers ${ }^{a}$ & $\begin{array}{l}48.6 \% \\
(570)\end{array}$ & $\begin{array}{c}35.0 \% \\
(243)\end{array}$ & $\begin{array}{l}68.3 \% \\
(327)\end{array}$ \\
\hline Active smoker & $\begin{array}{l}14.8 \% \\
(174)\end{array}$ & $\begin{array}{c}71.8 \% \\
(124)\end{array}$ & $\begin{array}{c}10.4 \% \\
(50)\end{array}$ \\
\hline Former smoker & $\begin{array}{c}36.6 \% \\
(430)\end{array}$ & $\begin{array}{l}47.2 \% \\
(328)\end{array}$ & $\begin{array}{c}21.3 \% \\
(102)\end{array}$ \\
\hline Relative's history of AAA & $\begin{array}{l}3.0 \% \\
(35) *\end{array}$ & $\begin{array}{c}2.3 \% \\
(16)\end{array}$ & $\begin{array}{c}4 \% \\
(19)^{*}\end{array}$ \\
\hline First degree & $\begin{array}{c}74.3 \% \\
(26)\end{array}$ & $\begin{array}{c}87.5 \% \\
(14)\end{array}$ & $\begin{array}{c}63.2 \% \\
(12)\end{array}$ \\
\hline Second degree & $\begin{array}{c}14.3 \% \\
(5)\end{array}$ & $\begin{array}{c}12.5 \% \\
(2)\end{array}$ & $\begin{array}{c}15.8 \% \\
\text { (3) }\end{array}$ \\
\hline Diabetes mellitus ${ }^{a}$ & $\begin{array}{l}17.7 \% \\
(208)\end{array}$ & $\begin{array}{c}23.2 \% \\
(161)\end{array}$ & $\begin{array}{c}9.8 \% \\
(47)\end{array}$ \\
\hline Dyslipidemia & $\begin{array}{l}46.3 \% \\
(544)\end{array}$ & $\begin{array}{l}45.9 \% \\
(319)\end{array}$ & $\begin{array}{c}47 \% \\
(225)\end{array}$ \\
\hline Hypertension $^{a}$ & $\begin{array}{l}47.8 \% \\
(561)\end{array}$ & $\begin{array}{c}52.9 \% \\
(368)\end{array}$ & $\begin{array}{l}40.3 \% \\
(193)\end{array}$ \\
\hline Chronic renal disease $^{\mathrm{a}}$ & $\begin{array}{c}4.1 \% \\
(48)\end{array}$ & $\begin{array}{c}5.8 \% \\
(40)\end{array}$ & $\begin{array}{c}1.7 \% \\
(8)\end{array}$ \\
\hline
\end{tabular}


Table 4. Cont.

\begin{tabular}{|c|c|c|c|}
\hline & $\begin{array}{c}\text { Total } \\
(n=1174)\end{array}$ & $\begin{array}{c}\text { Male } \\
(n=695)\end{array}$ & $\begin{array}{c}\text { Female } \\
(n=479)\end{array}$ \\
\hline \multicolumn{4}{|c|}{ Cardiovascular events \% (N) } \\
\hline Cardiac ischemia $^{\text {a }}$ & $\begin{array}{l}6.4 \% \\
(75)\end{array}$ & $\begin{array}{c}9.8 \% \\
(68)\end{array}$ & $\begin{array}{c}1.5 \% \\
(7)\end{array}$ \\
\hline $\begin{array}{c}\text { Cerebrovascular } \\
\text { events }\end{array}$ & $\begin{array}{l}4.3 \% \\
(50)\end{array}$ & $\begin{array}{l}4.9 \% \\
(34)\end{array}$ & $\begin{array}{l}3.3 \% \\
(16)\end{array}$ \\
\hline Intermittent claudication & $\begin{array}{l}3.8 \% \\
(45)\end{array}$ & $\begin{array}{l}4.5 \% \\
(31)\end{array}$ & $\begin{array}{l}2.9 \% \\
(14)\end{array}$ \\
\hline \multicolumn{4}{|c|}{ Anthropometric measurements \% (N) } \\
\hline Waist circumference $(\mathrm{cm})^{\mathrm{b}}(\mathrm{SD})$ & $\begin{array}{l}99.5 \\
(12)\end{array}$ & $\begin{array}{l}102.4 \\
(10.9)\end{array}$ & $\begin{array}{c}95.3 \\
(12.3)\end{array}$ \\
\hline Mean BMI $\left(\mathrm{kg} / \mathrm{m}^{2}\right)(\mathrm{SD})$ & $\begin{array}{l}27.7 \\
(4.3)\end{array}$ & $\begin{array}{l}27.8 \\
(3.8)\end{array}$ & $\begin{array}{l}27.6 \\
(4.9)\end{array}$ \\
\hline $\begin{array}{l}\text { Normal weight }{ }^{\text {a }} \\
\text { (BMI 18.5-24.9) }\end{array}$ & $\begin{array}{c}28.3 \% \\
(332)\end{array}$ & $\begin{array}{c}24.2 \% \\
(168)\end{array}$ & $\begin{array}{l}34.2 \% \\
(164)\end{array}$ \\
\hline $\begin{array}{l}\text { Overweight } \\
\text { (BMI 25-30) }\end{array}$ & $\begin{array}{l}46.3 \% \\
(543)\end{array}$ & $\begin{array}{l}51.4 \% \\
(357)\end{array}$ & $\begin{array}{l}38.8 \% \\
(186)\end{array}$ \\
\hline Obesity $(\mathrm{BMI}>30)$ & $\begin{array}{l}25.5 \% \\
(299)\end{array}$ & $\begin{array}{l}24.5 \% \\
(170)\end{array}$ & $\begin{array}{l}26.9 \% \\
(129)\end{array}$ \\
\hline
\end{tabular}

* 4 of these, unknown degree; ${ }^{\mathrm{a}} p<0.001$ Pearson's chi-square; $^{\mathrm{b}} p<0.001$ Student's $t$-test; Percentage (n). Mean and standard deviation.

Characteristics of our participants with those obtained from the 2018 Catalan Health Survey "Enquesta Catalunya de Salut 2018" (ESCA) and primary care centers located in our area of treatment of Barcelona Nord were reviewed in order to better assess the representation of our sample participants with our overall area of treatment population, as shown in Table 5.

Table 5. Characteristics of our study participants and participants of Catalunya Health Survey 2018 (ESCA) and primary care centers of our region of study separated by sex.

\begin{tabular}{|c|c|c|c|c|c|c|c|c|c|}
\hline & \multicolumn{3}{|c|}{$\begin{array}{l}\text { Our Study Participants } \\
\text { (65 Years Old) }\end{array}$} & \multicolumn{3}{|c|}{$\begin{array}{c}\text { ESCA } 2018 \\
\text { (65-74 Years Old) }\end{array}$} & \multicolumn{3}{|c|}{$\begin{array}{c}\text { Primary Care Centers } \\
\text { (65 Years Old) }\end{array}$} \\
\hline & $\begin{array}{c}\text { Male } \\
(n=695)\end{array}$ & $\begin{array}{l}\text { Female } \\
(n=479)\end{array}$ & $\begin{array}{c}\text { Total } \\
(n=1174)\end{array}$ & $\begin{array}{c}\text { Male } \\
(n=206)\end{array}$ & $\begin{array}{c}\text { Female } \\
(n=224)\end{array}$ & $\begin{array}{c}\text { Total } \\
(n=430)\end{array}$ & $\begin{array}{c}\text { Male } \\
(n=1653)\end{array}$ & $\begin{array}{c}\text { Female } \\
(n=1994)\end{array}$ & $\begin{array}{c}\text { Total } \\
(n=3647)\end{array}$ \\
\hline $\begin{array}{l}\text { Active } \\
\text { smoker }\end{array}$ & $\begin{array}{c}17.8 \% \\
(15.0-20.7)\end{array}$ & $\begin{array}{c}10.4 \% \\
(7.7-13.2)\end{array}$ & $\begin{array}{c}14.8 \% \\
(12.8-16.9)\end{array}$ & $\begin{array}{c}18.0 \% \\
(12.7-23.2)\end{array}$ & $\begin{array}{c}8.0 \% \\
(4.5-11.6)\end{array}$ & $\begin{array}{c}12.8 \% \\
(4.5-11.6)\end{array}$ & $\begin{array}{c}20.8 \% \\
(18.8-22.7)\end{array}$ & $\begin{array}{c}13.4 \% \\
(11.9-14.9)\end{array}$ & $\begin{array}{c}16.8 \% \\
(15.5-17.9)\end{array}$ \\
\hline $\begin{array}{l}\text { Diabetes } \\
\text { mellitus }\end{array}$ & $\begin{array}{c}23.2 \% \\
(20.0-26.3)\end{array}$ & $\begin{array}{c}9.8 \% \\
(7.2-12.5)\end{array}$ & $\begin{array}{c}17.7 \% \\
(15.5-19.9)\end{array}$ & $\begin{array}{c}27.2 \% \\
(21.1-33.3)\end{array}$ & $\begin{array}{c}20.1 \% * \\
(14.8-25.3)\end{array}$ & $\begin{array}{c}23.5 \% * \\
(19.5-27.5)\end{array}$ & $\begin{array}{c}23.1 \% \\
(21.0-25.1)\end{array}$ & $\begin{array}{c}12.8 \% * \\
(11.4-14.3)\end{array}$ & $\begin{array}{c}17.5 \% \\
(16.2-18.7)\end{array}$ \\
\hline Dyslipidemia & $\begin{array}{c}45.0 \% * \\
(42.2-49.6)\end{array}$ & $\begin{array}{c}47.0 \% \\
(42.5-51.4)\end{array}$ & $\begin{array}{c}46.3 \% \\
(43.5-49.2)\end{array}$ & $\begin{array}{c}34.5 \% * \\
(28.0-41.0)\end{array}$ & $\begin{array}{c}46.0 \% \\
(39.5-52.5)\end{array}$ & $\begin{array}{c}40.5 \% * \\
(35.8-45.1)\end{array}$ & $\begin{array}{c}39.4 \% * \\
(37.1-41.8)\end{array}$ & $\begin{array}{c}41.7 \% * \\
(39.5-43.8)\end{array}$ & $\begin{array}{c}40.7 \% * \\
(39.1-42.3)\end{array}$ \\
\hline Hypertension & $\begin{array}{c}53.0 \% \\
(49.2-56.7) \\
\end{array}$ & $\begin{array}{c}40.3 \% \\
(35.9-44.7) \\
\end{array}$ & $\begin{array}{c}47.8 \% \\
(44.9-50.6) \\
\end{array}$ & $\begin{array}{c}50.5 \% \\
(43.7-57.3) \\
\end{array}$ & $\begin{array}{c}48.2 \% * \\
(41.7-54.8) \\
\end{array}$ & $\begin{array}{c}49.3 \% \\
(44.6-54.0) \\
\end{array}$ & $\begin{array}{c}53.8 \% \\
(51.4-56.2) \\
\end{array}$ & $\begin{array}{c}62.9 \% * \\
(60.8-65.1)\end{array}$ & $\begin{array}{c}58.8 \% * \\
(57.2-60.4)\end{array}$ \\
\hline $\begin{array}{l}\text { Chronic } \\
\text { renal disease }\end{array}$ & $\begin{array}{c}5.8 \% \\
(4.0-7.5)\end{array}$ & $\begin{array}{c}1.7 \% \\
(0.5-2.8)\end{array}$ & $\begin{array}{c}4.1 \% \\
(3.0-5.2)\end{array}$ & $\begin{array}{c}5.8 \% \\
(2.6-9.0)\end{array}$ & $\begin{array}{c}6.3 \% * \\
(3.1-9.4)\end{array}$ & $\begin{array}{c}6.1 \% * \\
(3.8-8.3)\end{array}$ & $\begin{array}{c}4.1 \% \\
(3.1-5.0)\end{array}$ & $\begin{array}{c}2.3 \% \\
(1.6-2.9)\end{array}$ & $\begin{array}{c}3.1 \% \\
(2.5-3.6)\end{array}$ \\
\hline $\begin{array}{l}\text { Cardiac } \\
\text { ischemia }\end{array}$ & $\begin{array}{c}9.8 \% \\
(7.6-12.0)\end{array}$ & $\begin{array}{c}1.5 \% \\
(0.4-2.5)\end{array}$ & $\begin{array}{c}6.4 \% \\
(5.0-7.8)\end{array}$ & $\begin{array}{c}7.3 \% \\
(3.7-10.8)\end{array}$ & $\begin{array}{c}2.2 \% \\
(0.3-4.2)\end{array}$ & $\begin{array}{c}4.7 \% \\
(2.7-6.6)\end{array}$ & $\begin{array}{c}8.1 \% \\
(6.8-9.4)\end{array}$ & $\begin{array}{c}1.8 \% \\
(1.2-2.4)\end{array}$ & $\begin{array}{c}4.7 \% \text { * } \\
(3.9-5.4)\end{array}$ \\
\hline $\begin{array}{l}\text { Cerebrovascular } \\
\text { events }\end{array}$ & $\begin{array}{c}4.9 \% \\
(3.3-6.5) \\
\end{array}$ & $\begin{array}{c}3.3 \% \\
(1.7-5.0) \\
\end{array}$ & $\begin{array}{c}4.3 \% \\
(3.1-5.4)\end{array}$ & $\begin{array}{c}5.8 \% \\
(2.6-9.0) \\
\end{array}$ & $\begin{array}{c}2.7 \% \\
(0.6-4.8) \\
\end{array}$ & $\begin{array}{c}4.2 \% \\
(2.3-6.1) \\
\end{array}$ & $\begin{array}{c}3.1 \% \\
(2.3-3.9) \\
\end{array}$ & $\begin{array}{c}1.4 \% \\
(0.9-1.9) \\
\end{array}$ & $\begin{array}{c}2.1 \%{ }^{*} \\
(1.7-2.6) \\
\end{array}$ \\
\hline $\begin{array}{l}\text { Overweight } \\
\text { (BMI 25-30) }\end{array}$ & $\begin{array}{c}51.4 \% \\
(47.7-55.1) \\
\end{array}$ & $\begin{array}{c}38.8 \% \\
(34.5-43.2)\end{array}$ & $\begin{array}{c}46.3 \% \\
(43.4-49.1)\end{array}$ & $\begin{array}{c}46.6 \% \\
(39.8-53.4) \\
\end{array}$ & $\begin{array}{c}42.0 \% \\
(35.5-48.4)\end{array}$ & $\begin{array}{c}44.2 \% \\
(39.5-48.9) \\
\end{array}$ & $\begin{array}{c}38.2 \% * \\
(35.8-40.5)\end{array}$ & $\begin{array}{c}30.9 \% * \\
(28.9-33.0)\end{array}$ & $\begin{array}{c}34.2 \% \text { * } \\
(32.7-35.8)\end{array}$ \\
\hline $\begin{array}{c}\text { Obesity } \\
(\mathrm{BMI}>30)\end{array}$ & $\begin{array}{c}24.5 \% \\
(21.3-27.7)\end{array}$ & $\begin{array}{c}26.9 \% \\
(23.0-31.0)\end{array}$ & $\begin{array}{c}25.5 \% \\
(23.0-28.0)\end{array}$ & $\begin{array}{c}27.2 \% \\
(21.2-33.3)\end{array}$ & $\begin{array}{c}17.4 \% * \\
(12.4-22.4)\end{array}$ & $\begin{array}{c}22.1 \% \\
(18.2-26.0)\end{array}$ & $\begin{array}{c}27.7 \% \\
(25.6-29.7)\end{array}$ & $\begin{array}{c}29.5 \% \\
(27.5-31.5)\end{array}$ & $\begin{array}{c}28.7 \% \text { * } \\
(27.2-30.2)\end{array}$ \\
\hline
\end{tabular}

Values are presented as percentages (95\% Confidence Interval); Pearson's chi-square analysis; ${ }^{*}$ Difference in values among both studies. 


\section{Discussion}

In the present study, the prevalence of PAD in asymptomatic patients was $6.2 \%$ at age 65, which is in accordance with previous studies carried out in the Mediterranean area $[13,14,17]$. Risk factors are commonly known to be associated with other atherosclerotic diseases, with predominant factors being those of active smoking and diabetes [14]. This was shown to be similar in our study, with associated risk factors being smoking, history of diabetes, cardiac ischemia and a large waist circumference, smoking being the strongest association. Independent risk factors were also seen in logistic regression analysis in male smokers who had a history of diabetes and in female smokers who had hypertension. Knowing the important association of PAD with high cardiovascular risk groups, nonscreening in these subjects could lead to an underdiagnosis or even underestimate the true cardiovascular risk of this disease, outlining the importance of screening in these subgroups.

\subsection{Differences According to Geography and Age}

The worldwide prevalence of PAD is estimated to be 3-12\% [18], affecting 27 million people in America and Europe [19]. In Europe, the prevalence of PAD is estimated at around $17.8 \%$ (99\% CI: $16.8-18.8 \%$ ) in the ages of 45 and 55, seen in the PANDORA study [10].

In Spain, however, the prevalence of PAD is considerably lower. The ESTIME study [20] estimated an $8.03 \%$ prevalence in similar age groups as the PANDORA study [10] in Europe, and studies published in the Catalonia Region of Spain concur with these low results. In this sense, Velescu et al. [14] reported a prevalence as low as $4.5 \%$ in Girona, and the Perart/Artper group of Barcelona reported a 7.6\% prevalence [17]. Although both results are relatively much lower than estimated in Europe and the USA, the observed difference amongst the two might be due in part to the difference in the studied age group, with the Velescu group including subjects as young as 35 years old. This demonstrates the increase in prevalence with age that has already been described previously and the variability of prevalence with geographic regions due to ethnicity and dietary lifestyle, which tend to be unique to each region of study [21,22].

\subsection{Sex Differences}

Traditionally PAD is thought to be a male-predominant disease [23], but in recent studies, this has been proven otherwise, with an increase of prevalence in elderly women. About $20-30 \%$ of women over 70 years old are affected by PAD [24]. Peripheral arterial disease is frequently underdiagnosed due to atypical symptoms and its late presentation in females, and some studies even suggest a 10 to 12 years delay in appearance. Our prevalence of PAD in women was $3.8 \%$ compared to $7.9 \%$ in males, perhaps partly, again, due to the age group selection, needing further studies to observe prevalence in older female groups.

\subsection{Risk Factors}

The association of PAD with coronary artery disease is observed in $50 \%$ of patients and in $20 \%$ of patients with cerebrovascular disease $[25,26]$, becoming therefore an important indicator of the coexistence of other cardiovascular disorders [27]. Up to $40 \%$ of subjects with PAD die from coronary artery disease and $10-20 \%$ by cerebral artery disease [10]. This risk is not only high in asymptomatic subjects but has a straight relationship among symptomatic individuals and severe PAD measured through ABI [28]. Overall, only less than $40 \%$ of subjects with PAD do not have concomitant coronary or cerebrovascular disease. Therefore, the association of PAD with other cardiovascular diseases is one of the main concerns of this disease. In our study, $13.6 \%$ of our subjects with PAD had an associated history of ischemic heart disease, and only a small few had a history of ischemic cerebrovascular disease.

The strongest predictor of PAD in those 65 years and younger has been associated with diabetes and smoking [3], although our study concurs with this from the male sex 
perspective. This was different in our study for females. After logistic regression analysis, we found an independent risk factor association between women who were smokers and had hypertension ORs of 3.3 (95\% CI: 1.2-9.0) and 5.2 (95\% CI: 1.6-17.3), showing a stronger association between smoking and hypertension than with diabetes in our female group.

Stratification of cardiovascular risk was also attempted through the REGICOR score to identify those who had a higher risk of developing a cardiovascular event in the following 10 years. Altogether, we found higher scores in males $(p<0.001)$ with a high-risk score (considered $>10 \%$ ) in those with PAD at $14.8 \%$ versus those with non-PAD at $12.7 \%$. Therefore, screening with $\mathrm{ABI}$ in these subjects we believe to be important for the early implementation of secondary cardiovascular prevention.

\subsection{Limitations}

It should be noted that this study is part of a larger pilot screening program evaluating abdominal aorta aneurysms [8], thus explaining the chosen method of selection. Nonetheless, in order to minimize any selection bias that could affect our results, we reviewed our study population with the 2018 Catalan Health Survey "Enquesta Catalunya de Salut 2018" (ESCA) [29], focusing on the population age group, 65-74 years of age, and to the data obtained from our primary care centers in 2018, focusing on 65 years of age. We looked into various risk factors among our participants and those of ESCA and our primary care centers shown previously. In general, our participants are similar, although some differences are to be expected due to the wider age group of the survey and different methods of obtaining participant data. All this could lead to some variations seen in our sample participants. Due to limitations in our resources, we were forced to focus on one specific age group in our study. Additionally, our participation rate was $43.1 \%$, which is consistent with the participation rate of other screening programs performed in our region that have a wider broadcasting and media diffusion such as colon and breast cancer screening in Barcelona, which achieved participation rates of $43.6 \%$ and $54.7 \%$, respectively [30]. Overall, we believe this is a correct representation of our population, although these limitations should be noted.

\section{Conclusions}

In summary, the overall prevalence of PAD in North Barcelona at 65 years old is still significantly low compared to other regions of Europe [10], and male subjects were still predominantly affected by this disease than females at this age.

Our results concur with the literature on this topic for our region $[14,17]$ and outline the strong association between male smokers with diabetes and female smokers with hypertension, stressing the importance of our focus on these subgroups and perhaps emphasizing the need for ABI screening programs, as well as implementing secondary prevention if PAD is confirmed in these specific group of subjects. Additional follow-up studies would be interesting to further evaluate our initial results and differences in sex, as well as prevalence in older populations.

Author Contributions: Conceptualization, S.B.-M., G.G.-M. and J.F.; methodology, S.B.-M., G.G.-M. and J.F.; software, S.B.-M., T.P. and R.G.; formal analysis, T.P.; investigation, G.G.-M., S.B.-M., T.P. and R.G.; resources, S.B.-M.; data curation, G.G.-M., D.G.-S., C.T.-D., X.T.-S. and C.M.; writingoriginal draft preparation, G.G.-M. and S.B.-M.; writing—review and editing, G.G.-M., S.B.-M., T.P., R.G., and D.G.-S.; supervision, S.B.-M., T.P. and R.G.; project administration, S.B.-M., J.F. and T.P.; funding acquisition, S.B.-M. and J.F. All authors have read and agreed to the published version of the manuscript.

Funding: This work was logistically supported by the PERIS 2016-2020 medical research grant from Generalitat de Catalunya (Spain). Expedient Number SLT002/16/00441.

Institutional Review Board Statement: The study was conducted according to the guidelines of the Declaration of Helsinki and approved by the Ethics Committee of Vall d'Hebron University Hospital (PR(AG)221/2017). 
Informed Consent Statement: Informed consent was obtained of all subjects involved in the study.

Data Availability Statement: Not applicable.

Acknowledgments: A special thanks to Manuel Quintana Luque (Statistic Department of Hospital Vall d' Hebron), Judit Solà Roca (Clinical Epidemiology and Public Health Department of Hospital de la Santa Creu i Sant Pau) as well as Darío Gómez and Olga Solà for their help and support of this study.

Conflicts of Interest: The authors declare no conflict of interest.

$\begin{array}{ll}\text { Abbreviations } \\ \text { PAD } & \text { peripheral arterial disease } \\ \text { ABI } & \text { ankle-brachial index } \\ \text { OR } & \text { odds ratio } \\ \text { CI } & \text { confidence interval } \\ \text { AAA } & \text { abdominal aortic aneurysm } \\ \text { BMI } & \text { body mass index }\left(\mathrm{kg} / \mathrm{m}^{2}\right) \\ \text { SD } & \text { standard deviation }\end{array}$

\section{References}

1. Aronow, W.S. Peripheral arterial disease of the lower extremities. Arch. Med. Sci. 2012, 8, 375-388. [CrossRef]

2. Fowkes, F.G.; Aboyans, V.; Fowkes, F.J.; McDermott, M.M.; Sampson, U.K.; Criqui, M.H. Peripheral artery disease: Epidemiology and global perspectives. Nat. Rev. Cardiol. 2017, 14, 156-170. [CrossRef]

3. Criqui, M.H.; Aboyans, V. Epidemiology of Peripheral Artery Disease. Circ. Res. 2015, 116, 1509-1526. [CrossRef]

4. Hirsch, A.T.; Haskal, Z.J.; Hertzer, N.R.; Bakal, C.W.; Creager, M.A.; Halperin, J.L.; Hiratzka, L.F.; Murphy, W.R.; Olin, J.W.; Puschett, J.B.; et al. ACC/AHA 2005 Practice Guidelines for the Management of Patients With Peripheral Arterial Disease (Lower Extremity, Renal, Mesenteric, and Abdominal Aortic). Circulation 2006, 113, e463-e654. [CrossRef]

5. Hirsch, A.T.; Criqui, M.H.; Treat-Jacobson, D.; Regensteiner, J.G.; Creager, M.A.; Olin, J.W.; Krook, S.H.; Hunninghake, D.B.; Comerota, A.J.; Walsh, M.E.; et al. Peripheral Arterial Disease Detection, Awareness, and Treatment in Primary Care. JAMA 2001, 286, 1317-1324. [CrossRef]

6. Aboyans, V.; Ricco, J.B.; Bartelink, M.L.E.; Björck, M.; Brodmann, M.; Cohnert, T.; Desormais, I. 2017 ESC Guidelines on the Diagnosis and Treatment of Peripheral Arterial Diseases, in Collaboration With the European Society for Vascular Surgery (ESVS): Document Covering Atherosclerotic Disease of Extracranial Carotid and Vertebral, Mesenteric, Renal, Upper and Lower Extremity arteries Endorsed By: The European Stroke Organization (ESO) The Task Force for the Diagnosis and Treatment of Peripheral Arterial Diseases of the European Society of Cardiology (ESC) and of the European Society for Vascular Surgery (ESVS). Eur. Heart J. 2018, 39, e35-e41.

7. Aboyans, V.; Criqui, M.H.; Abraham, P.; Allison, M.A.; Creager, M.A.; Diehm, C.; American Heart Association Council on peripheral Vascular Disease; Council on Epidemiology and prevention; Council on clinical Cardiology; Council on Cardiovascular nursing; et al. Measurement and interpretation of the ankle-brachial index: A scientific statement from the American Heart Association. Circulation 2012, 126, 2890-2909. [CrossRef]

8. Benjamin, E.J.; Virani, S.S.; Callaway, C.W.; Chamberlain, A.M.; Chang, A.R.; Cheng, S.; Muntner, P.; American Heart Association Council on Epidemiology; Prevention Statistics Committee; Stroke Statistics Subcommittee. Heart Disease and Stroke Statistics2018 Update: A Report from the American Heart Association. Circulation 2018, 137, e67-e492. [CrossRef]

9. Firnhaber, J.M.; Powell, C.S. Lower Extremity Peripheral Artery Disease: Diagnosis and Treatment. Am. Fam. Physician 2019, 99, 362-369.

10. Cimminiello, C.; Kownator, S.; Wautrecht, J.C.; Carvounis, C.P.; Kranendonk, S.E.; Kindler, B.; Borghi, C. The PANDORA study: Peripheral arterial disease in patients with non-high cardiovascular risk. Intern. Emerg. Med. 2011, 6, 509-519. [CrossRef]

11. SSchramm, K.; Rochon, P.J. Gender Differences in Peripheral Vascular Disease. Semin. Interv. Radiol. 2018, 35, 009-016. [CrossRef]

12. Norgren, L.; Hiatt, W.R.; Dormandy, J.A.; Nehler, M.R.; Harris, K.A.; Fowkes, F.G. Inter-Society Consensus for the Management of Peripheral Arterial Disease (TASC II). J. Vasc. Surg. 2007, 45, S5-S67. [CrossRef]

13. Ramos, R.; Quesada, M.; Solanas, P.; Subirana, I.; Sala, J.; Vila-Domènech, J.S.; Masiá, R.; Cerezo, C.; Elosua, R.; Grau, M.; et al. Prevalence of Symptomatic and Asymptomatic Peripheral Arterial Disease and the Value of the Ankle-brachial Index to Stratify Cardiovascular Risk. Eur. J. Vasc. Endovasc. Surg. 2009, 38, 305-311. [CrossRef]

14. Velescu, A.; Clara, A.; Peñafiel, J.; Grau, M.; Degano, I.R.; Martí, R.; Ramos, R.; Marrugat, J.; Elosua, R. Peripheral Arterial Disease Incidence and Associated Risk Factors in a Mediterranean Population-based Cohort. The REGICOR Study. Eur. J. Vasc. Endovasc. Surg. 2016, 51, 696-705. [CrossRef] 
15. Fite, J.; Gayarre-Aguado, R.; Puig, T.; Zamora, S.; Escudero, J.R.; Solà Roca, J.; Bellmunt-Montoya, S. Feasibility and Efficiency Study of a Population-Based Abdominal Aortic Aneurysm Screening Program in Men and Women in Spain. Ann. Vasc. Surg. 2021, 73, 429-437. [CrossRef]

16. Amor, A.J.; Serra-Mir, M.; Martínez-González, M.A.; Corella, D.; Salas-Salvad, J.; Fit, M.; Francisco, S. PREDIMED Investigators. Prediction of Cardiovascular Disease by the Framingham-REGICOR Equation in the High-Risk PREDIMED Cohort: Impact of the Mediterranean Diet across Different Risk Strata. J. Am. Heart Assoc. 2017, 6, e004803. [CrossRef]

17. Alzamora, M.T.; Forés, R.; Baena-Díez, J.M.; Pera, G.; Toran, P.; Sorribes, M.; Llussà, J. The Peripheral Arterial disease study (PERART/ARTPER): Prevalence and risk factors in the general population. BMC Public Health 2010, 10, 1-11. [CrossRef]

18. Harris, L.; Dryjski, M. Epidemiology, Risk Factors and Natural History of Lower Extremity Peripheral Artery Disease. UpToDate 2020, 23, 2020. Available online: https:/ / www.uptodate.com/contents/epidemiology-risk-factors-and-natural-history-of-lowerextremity-peripheral-artery-disease (accessed on 15 January 2020).

19. Suárez, C.; Lozano, F.S.; Bellmunt, S.; Camafort, M.; Díaz, S.; Mancera, J.; Carrasco, E.; Lobos, J.M. Documento de Consenso Multidisciplinar en Torno a la Enfermedad Arterial Periférica, 1st ed.; Luzán 5, S.A.: Madrid, Spain, 2012.

20. Blanes, J.I.; Cairols, M.A.; Marrugat, J. Prevalence of peripheral artery disease and its associated risk factors in Spain: The ESTIME Study. Int. Angiol. 2009, 28, 20-25.

21. Dontas, A.S.; Zerefos, N.S.; Panagiotakos, D.B.; Vlachou, C.; Valis, D.A. Medieterranean diet and prevention of coronary heart disease in the elderly. Clin. Interv. Aging. 2007, 2, 109. [CrossRef]

22. McDermott, M.M.; Liu, K.; Criqui, M.H.; Ruth, K.; Goff, D.; Saad, M.H.; Sharrett, A.R. Ankle-Brachial Index and subclinical cardiac and carotid disease: The multi-ethnic study of atherosclerosis. Am. J. Epidemiol. 2005, 162, 33-41. [CrossRef]

23. Parvar, S.L.; Thiyagarajah, A.; Nerlekar, N.; King, P.; Nicholls, S.J. A systematic review and meta-analysis of gender differences in long-term mortality and cardiovascular events in peripheral artery disease. J. Vasc. Surg. 2021, 73, 1456-1465. [CrossRef]

24. Criqui, M.H.; Fronek, A.; Barrett-Connor, E.; Klauber, M.R.; Gabriel, S.; Goodman, D. The prevalence of peripheral arterial disease in a defined population. Circulation 1985, 71, 510-515. [CrossRef]

25. Fowkes, F.G.R.; Housley, E.; Cawood, E.H.H.; Macintyre, C.C.A.; Ruckley, C.V.; Prescott, R.J. Edinburgh Artery Study: Prevalence of Asymptomatic and Symptomatic Peripheral Arterial Disease in the General Population. Int. J. Epidemiol. 1991, $20,384-392$. [CrossRef]

26. Olinic, D.M.; Spinu, M.; Olinic, M.; Homorodean, C.; Tataru, D.A.; Liew, A.; Catalano, M. Epidemiology of peripheral artery disease in Europe: VAS Educational Paper. Int. Angiol. 2018, 37, 327-334. [CrossRef]

27. Venermo, M.; Sprynger, M.; Desormais, I.; Björck, M.; Brodmann, M.; Cohnert, T.; De Carlo, M.; Espinola-Klein, C.; Kownator, S.; Mazzolai, L.; et al. Editor's Choice-Follow-up of Patients After Revascularisation for Peripheral Arterial Diseases: A Consensus Document From the European Society of Cardiology Working Group on Aorta and Peripheral Vascular Diseases and the European Society for Vascular Surgery. Eur. J. Vasc. Endovasc. Surg. 2019, 58, 641-653.

28. Newman, A.B.; Shemanski, L.; Manolio, T.A.; Cushman, M.; Mittelmark, M.; Polak, J.F.; Siscovick, D. Ankle-arm index as a predictor of cardiovascular disease and mortality in the Cardiovascular Health Study. Arter. Thromb. Vasc. Biol. 1999, 19, 538-545. [CrossRef] [PubMed]

29. Resultats de L'enquesta de Salut de Catalunya (ESCA). Departament De Salut, Generalitat De Catalunya. Available online: https: //salutweb.gencat.cat/ca/el_departament/estadistiques_sanitaries/enquestes/esca/resultats_enquesta_salut_catalunya/ (accessed on 2 February 2021).

30. Burón, A.; Grau, J.; Andreu, M.; Augé, J.M.; Guayta-Escolies, R.; Barau, M.; Castells, A. Programa de Detección Precoz de Cáncer de Colon y Recto de Barcelona: Indicadores de la primera ronda de un programa con participación de la farmacia comunitaria. Med. Clin. 2015, 145, 141-146. [CrossRef] 